\title{
Publisher Correction: Long-term functional and structural preservation of precision-cut human myocardium under continuous electromechanical stimulation in vitro
}

\author{
Carola Fischer ${ }^{1}$, Hendrik Milting ${ }^{2}$, Evelyn Fein ${ }^{1}$, Elisabeth Reiser ${ }^{1}$, Kun Luํ․ Thomas Seidel ${ }^{3}$, Camilla Schinner ${ }^{4}$, \\ Thomas Schwarzmayr ${ }^{5}$, Rene Schramm²,6, Roland Tomasi ${ }^{1,7}$, Britta Husse ${ }^{1}$, Xiaochun Cao-Ehlker ${ }^{1}$, \\ Ulrich Pohl ${ }^{1,8} \&$ Andreas Dendorfer ${ }^{1,8}$
}

Correction to: Nature Communications; https://doi.org/10.1038/s41467-018-08003-1, published online 10 January 2019

The original version of this Article incorrectly acknowledged Elisabeth Reiser and Rene Schramm as a corresponding author. This has now been corrected in both the PDF and HTML versions of the Article.

Published online: 28 January 2019

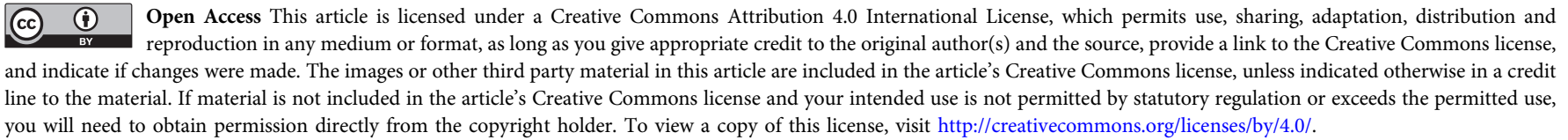
you will need to obtain permission directly from the copyright holder. To view a copy of this license, visit http://creativecommons.org/licenses/by/4.0/.

(c) The Author(s) 2019

\footnotetext{
${ }^{1}$ Walter-Brendel-Centre of Experimental Medicine, University Hospital, LMU Munich, Marchioninistr. 27, 81377 Munich, Germany. ${ }^{2}$ Erich \& Hanna Klessmann Institute, Clinic for Thoracic and Cardiovascular Surgery, Heart and Diabetes Center NRW, Ruhr-University Bochum, Georgstr. 11, 32545 Bad Oeynhausen, Germany. ${ }^{3}$ Institute of Cellular and Molecular Physiology, Friedrich-Alexander University Erlangen-Nürnberg, Waldstr. 8,91054 Erlangen, Germany. ${ }^{4}$ Institute of Anatomy, Ludwig-Maximilians-University Munich, Pettenkoferstr. 11, 80336 Munich," Germany. ${ }^{5}$ Institute of Human Genetics, Helmholtz Zentrum München, Ingolstädter Landstr. 1, 85764 Neuherberg, Germany. ${ }^{6}$ Clinic of Cardiac Surgery, University Hospital, LMU Munich, Marchioninistr. 15, 81377 Munich, Germany. ${ }^{7}$ Clinic of Anaesthesiology, University Hospital, LMU Munich, Marchioninistr. 15, 81377 Munich, Germany. ${ }^{8}$ German Center for Cardiovascular Research, partner site Munich Heart Alliance, 80802 Munich, Germany. Correspondence and requests for materials should be addressed to A.D. (email: andreas.dendorfer@med.uni-muenchen.de)
} 